\title{
Comparison of venlafaxine and duloxetine: measuring clinical impact of time to therapeutic dose (TTD) among patients achieving therapeutic dosing for pain
}

\begin{abstract}
Background: Chronic opioid therapy remains controversial; however, there is consensus among treatment guidelines that adjunct medications should be utilized first. A meta-analysis revealed serotonin-norepinephrine reuptake inhibitors (SNRIs) venlafaxine and duloxetine to be equally efficacious in the treatment of neuropathic pain and recommend them as first-line therapy.1 Although, the therapeutic dosing for pain with both medications is well established there are no head-to-head studies comparing the two medications. The impact of medication selection (TIMS) as measured by percentage of patients achieving therapeutic dose and time to therapeutic dose (TTD) for neuropathic pain with either venlafaxine or duloxetine is not well understood. These outcomes along with adverse effect profiles need to be evaluated to inform clinical decision making.
\end{abstract}

Materials and Methods: This was a single center, retrospective, observationa analysis. New start prescriptions for either venlafaxine or duloxetine between January 1, 2011 and January 1, 2014 were identified. Through data warehouse extraction the following was collected: age, gender, weight, height, race, comorbidities, prescriber and concomitant antidepressants and anticonvulsants on date of initiation. Manual data collection through the Computerized Patient Record System (CPRS) was then utilized to determine veteran eligibility as well as if therapeutic dose was achieved, TTD, as well as discontinuation rates and cause.

Results: 682 charts were reviewed to identify 302 patients, 151 in each group. The duloxetine group had 120 patients achieve therapeutic dose compared to 82 in the venlafaxine group $(\mathrm{p}<0.0001)$. Median TTD for duloxetine was 7 days $(0-44.25$, IQR) compared to venlafaxine 31.5 days (10-115, IQR). At study conclusion, 50/151 $(33.1 \%)$ patients remained on duloxetine compared to $31 / 151(20.5 \%)$ of those on venlafaxine (p-value 0.0191 ). Side effects were reported in $37 \%$ of patients in venlafaxine group compared to $22 \%$ of duloxetine group ( $\mathrm{p}=0.0053$ ). Of note, 117 $(77 \%)$ of the duloxetine patients had a previous trial of venlafaxine therapy.

Conclusion: Patients taking duloxetine are significantly more likely to achieve therapeutic dose, arrive at therapeutic dose more quickly, and remain on the medication compared to venlafaxine. Titration schedule may influence tolerability. Duloxetine should be favored over venlafaxine in treatment algorithms for neuropathic pain.

Keywords: antidepressants, duloxetine, venlafaxine, neuropathic pain, diabetic neuropathy, pain
Volume 6 Issue 2 - 2018

\author{
Timothy Atkinson,' Kelsie W Flynn, ${ }^{2}$ Jennifer \\ Baker $^{3}$ \\ 'Pain Management Director,VA Tennessee Valley Healthcare \\ System, USA \\ 2PGY-I Pharmacy Practice Resident, VA Tennessee Valley \\ Healthcare System, USA \\ ${ }^{3}$ Assistant Professor, College of Pharmacy, University of \\ Tennessee, USA
}

Correspondence: Timothy Atkinson, Clinical Pharmacy Specialist, Pain Management Director, PGY2 Pain Management \& Palliative Care Residency,VA Tennessee Valley Healthcare System, Murfreesboro, Tennessee, USA, EmailTimothy.Atkinson@va.gov

Received: March 09, 2018 | Published: April 17, 2018

\section{Introduction}

Managed Care and integrated health systems have obligations to control costs in their allocation of limited resources and utilize formulary management to balance cost savings concerns with available evidence. Pharmacoeconomic evaluations often decide the availability of therapies based purely on cost when no evidence exists for clinical superiority between two treatments. From a therapeutic perspective, this presents challenges when multiple medications are recommended as first-line options in available guidelines but no headto-head studies exist to establish superiority. The cost of randomized controlled trials (RCTs) to establish such superiority is prohibitive and therefore they are rarely performed. Randomized trials are also not typically reflective of clinical practice as they exclude higher risk or medically complex patients and do not last long enough to evaluate long-term efficacy, rate of discontinuations, and number of patients that remain on subtherapeutic doses. Patient's, however, do have the right to the most effective treatment and delaying or diverting treatment away from effective therapy for cost considerations has significant ethical considerations. Neuropathic pain is often a chronic, debilitating condition, with a complex pathophysiology. ${ }^{2}$ The American Academy of Pain Medicine highlighted a recent market research report which indicates that more than 1.5 billion people worldwide suffer from chronic pain and that approximately $3-4.5 \%$ of the global population suffers from neuropathic pain, with incidence rate increasing in the aging population. ${ }^{3}$ Current evidencebased treatment guidelines ${ }^{4,5}$ recommend the use of amitriptyline, duloxetine, venlafaxine, gabapentin, or pregabalin as first line therapy. 
Despite the efficacy of tricyclic antidepressants (TCAs) being largely established, they have moved out of favor due to their excessive side effects which include dry mouth, sedation, and blurred vision. ${ }^{5-7}$ Serotonin norepinephrine reuptake inhibitors (SNRIs) have largely replaced TCAs, working through the same pathway by inhibiting reuptake of norepinephrine, and are better tolerated. ${ }^{8}$ Venlafaxine and duloxetine, both SNRIs, have been shown to be effective in RCTs. ${ }^{9-11}$ Clinical trials have shown that neuropathic pain relief by SNRIs is dose dependent which is heavily influenced by receptor selectivity. Duloxetine is highly selective for norepinephrine with a receptor affinity $(10: 1)$ compared to venlafaxine (30:1), respectively. ${ }^{12}$ Therefore, venlafaxine requires a higher dose to reach analgesic therapeutic effect unlike duloxetine. This was reflected in clinical trials when efficacy for venlafaxine was shown at doses greater than $150 \mathrm{mg} /$ day and duloxetine $60 \mathrm{mg}$ daily. ${ }^{13,14}$ Literature assessing time to therapeutic effect of venlafaxine and duloxetine in the treatment of pain is limited to pharmaceutical trials; no head-to-head studies have been conducted. As venlafaxine and duloxetine have very different titration schedules, the difference in tolerability between medications depending on how quickly each is titrated to their therapeutic dose may play a large role in therapeutic success. In addition to percentage of patients achieving therapeutic dosing and time to therapeutic dose, the percentage of patients that remained on the medication would be very useful as long-term efficacy is not typically assessed in clinical trials. To address the above concerns we elected to perform a therapeutic impact of medication selection (TIMS) evaluation between venlafaxine and duloxetine which are both first-line options recommended for neuropathic pain.

\section{Methods}

This is a single center, retrospective, observational analysis that was conducted at the Tennessee Valley Healthcare System (TVHS), an integrated Veterans Affairs (VA) system consisting of two medical centers and several community-based outpatient clinics located throughout middle Tennessee and southern Kentucky. The study protocol was approved by TVHS Institutional Review Board (IRB) and exempted from patient notification because it was retrospective, observational, and de-identified. Using the Veterans Integrated Service Network 9 (VISN 9) data warehouse, an initial patient list was pulled identifying patients who were receiving care at TVHS between January 1, 2011 and January 1, 2014 with a new start prescription for either venlafaxine or duloxetine. Patient records that included previous trials of the same SNRI within a 5 year period and/or were being prescribed venlafaxine or duloxetine for an unspecified or nonpain related indication, were excluded. Additional data pulled included comorbidities, concomitant antidepressants by pharmacological class on date of initiation, active prescription for gabapentin or pregabalin on date of initiation, most recent eGFR at date of initiation, and patient demographics.

As time to therapeutic dose has not been assessed in a head to head study for these medications, we used discontinuation rates as a surrogate marker to determine minimum sample size needed. Based off of clinical trials this study required 302 patients, 151 patients per arm, to have at least $80 \%$ power allowing for detection of a difference of 15 percent increase or decrease of patient's titrated to therapeutic dose between venlafaxine and duloxetine treatment groups. After the patient list was compiled, manual data collection was utilized to determine if patients were eligible for inclusion. Patients were included in the analysis if they were $\geq 18$ years of age, eGFR $>30$, prescribed a new start prescription for venlafaxine or duloxetine with documented indication for pain within the specified time period. Patients were excluded if they had a previous trial of the same SNRI within a 5-year period and/or are being prescribed venlafaxine or duloxetine for unspecified or non-pain related indication. Those patients identified as fitting the inclusion criteria were then subject to a more extensive chart review. Starting dose and current dose were collected, as well as the difference in number of days from initiation to therapeutic dose or discontinuation. If SNRI prescription was discontinued or expired, chart notes were reviewed to determine cause.

All analyses were performed using the Excel or GraphPad Software systems. The Fishers Exacts test was used to compare rates of discontinuation, percent of veterans reaching therapeutic dose and all baseline demographics, except age. Age was assessed using the t-test. Nonparametric or non-continuous data is described using median and interquartile ranges and statistical analysis using MannWhitney U. Statistical significance was set at $p<0.05$ and all tests were 2-tailed (Figure 1).

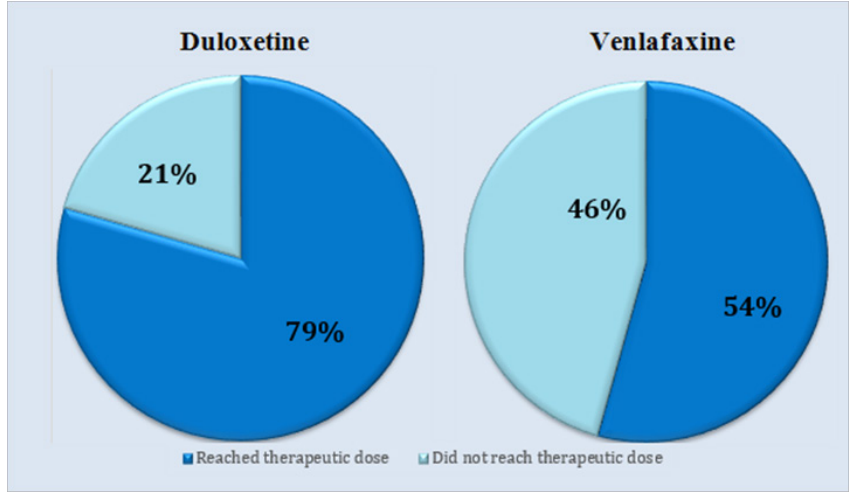

Figure 1 Percentage of patients achieving therapeutic dose.

\section{Results}

A total of 682 charts were reviewed with 302 patients meeting inclusion criteria, and 151 patients in each of the venlafaxine and duloxetine groups respectively. Baseline demographics were equivalent between groups, except for age, which was higher in the duloxetine group (55) versus venlafaxine group (48) and appears statistically significant ( $p$-value $<0.0001$ ). For the primary endpoint of percentage of patients achieving therapeutic doses (TD), the duloxetine group had 120/151 (79.5\%) versus the venlafaxine group which had $82 / 151(54.3 \%)$ (p-value $<0.0001)$. For the primary endpoint of time to $\mathrm{TD}$, the median time for duloxetine was 7 days (IQR 44 days) versus median time for venlafaxine was 31.5 days (IQR 105 days) (p-value 0.0001) (Figure 2).

For the secondary outcomes, the percentage discontinuation rate for duloxetine was 101/151 (66.9\%) with 50/120 (41.7\%) remaining on duloxetine at study conclusion compared to $120 / 151(79.5 \%)$ with $31 / 82(37.8 \%)$ remaining on venlafaxine. In absolute numbers, $50 / 151(33.1 \%)$ patients started on duloxetine remained on it at study conclusion compared to $31 / 151(20.5 \%)$ of those started on venlafaxine ( $\mathrm{p}$-value 0.0191$)$. The percentage discontinuation rate (Table 1) based on speed of titration for duloxetine versus venlafaxine showed, for $<14$ days to TD $64.5 \%$ compared to $87.5 \%$, for $14-30$ 
days to TD $55.6 \%$ compared to $80 \%$, for $30-60$ days to TD $55.6 \%$ compared to $90 \%$, for $60-90$ days to TD $25 \%$ compared to $50 \%$, and for $>90$ days to TD $63.6 \%$ compared to $48.3 \%$ (Figure 3). Reasons for discontinuation prior to achieving therapeutic dosing were primarily change in mood $(13.25 \%$ vs $9.27 \%)$, GI distress $(4.64 \%$ vs $4.64 \%)$, sexual dysfunction $(3.97 \%$ vs $2.65 \%)$, sedation $(2.65 \%$ vs $2.65 \%$ ), and nightmares $(2.65 \%$ vs $1.32 \%)$ for venlafaxine and duloxetine

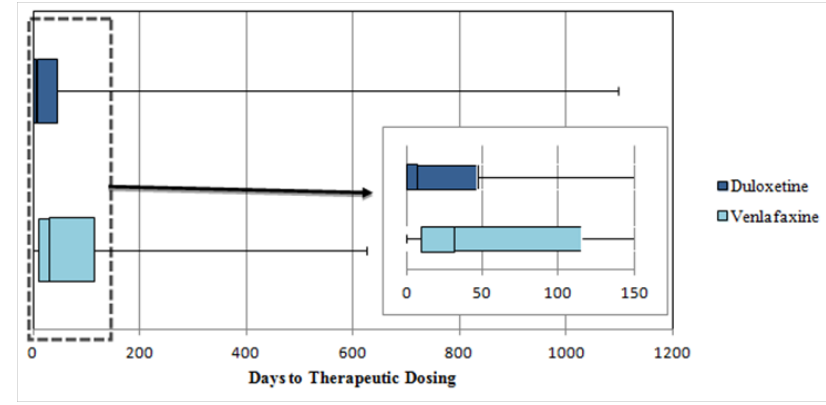

Figure 2 Time to therapeutic dose. respectively (Table 2). Concomitant diseases and medications between groups is represented in Table 3. Out of the 151 patients with new start prescriptions for duloxetine, $117(77.48 \%)$ tried venlafaxine prior to duloxetine. Of the 117 patients who trialed venlafaxine first, only 70 $(59.83 \%)$ achieved therapeutic dose. 35 of the 117 patients $(29.91 \%)$ received one prescription for venlafaxine before discontinuation (Table 1)(Figure 3).

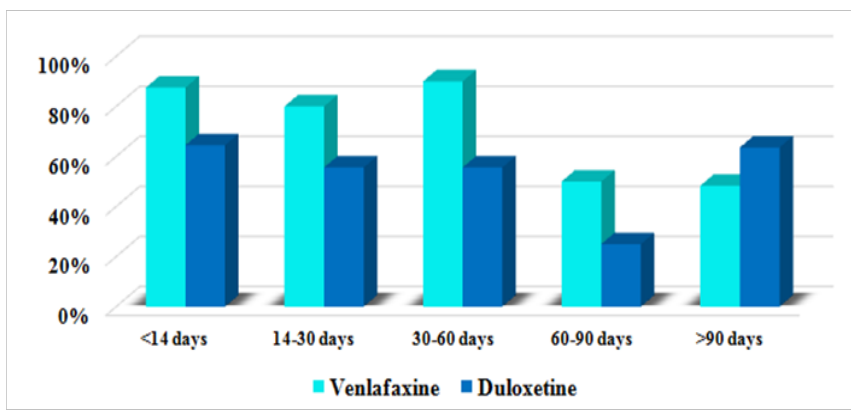

Figure 3 Influence of titration schedule on rate of discontinuation.

Table 1 Influence of time to therapeutic dose on discontinuation rate including side effects

\begin{tabular}{lllllll}
\hline Duloxetine & & & & & \\
\hline Time to reach TD: & Achieved & $\%$ & Discontinued/Expired & $\%$ & Caused by SE & $\%$ \\
\hline$<14$ days & 76 & 63.3 & 49 & 64.5 & 21 & 44.9 \\
$14-30$ days & 9 & 7.5 & 5 & 55.6 & 3 & 60 \\
$30-60$ days & 9 & 7.5 & 5 & 55.6 & 4 & 80 \\
$60-90$ days & 4 & 3.3 & 1 & 25 & 0 & 0 \\
$\geq 90$ days & 22 & 18.3 & 14 & 63.6 & 7 & 50 \\
\hline Venlafaxine & & & & & & \\
\hline Time to reach TD: & Achieved & $\%$ & Discontinued/Expired & $\%$ & Caused by SE & $\%$ \\
\hline$<14$ days & 24 & 29.3 & 21 & 87.5 & 14 & 66 \\
14-30 days & 15 & 18.3 & 12 & 80 & 7 & 58.3 \\
$30-60$ days & 10 & 12.2 & 9 & 90 & 6 & 66.7 \\
$60-90$ days & 4 & 4.9 & 2 & 50 & 1 & 50 \\
$\geq 90$ days & 29 & 35.4 & 14 & 48.3 & 5 & 35.7 \\
\hline
\end{tabular}

\section{Discussion}

Chronic opioid therapy remains controversial and its appropriateness, in chronic pain management, a subject of national debate. There is consensus, however, in treatment guidelines that adjunct medications should be utilized first and there is a sense of urgency to provide effective relief for patients in a timely manner. Lack of efficacy and intolerable side effects complicate treatment with adjunct medications and represent a significant source of frustration for patients. The therapeutic impact of medication selection (TIMS) is a critical concept to clinical practice and specifically pain management to determine which first-line therapy has the highest chance of efficacy, shortest time to therapeutic dose, and best long-term success. We applied this evaluation to use of venlafaxine and duloxetine for neuropathic pain (Table 2)(Table 3 ).

The percentage of patients achieving a therapeutic dose was selected as our primary outcome because it is clinically meaningful in pursuit of efficacy. The duloxetine group had significantly more veterans achieve therapeutic dosing 120 (79.5\%) versus 82/151 $(54.3 \%)$ in the venlafaxine group. However, of the 120 veterans in the duloxetine group that achieved a therapeutic dose, $70(59.3 \%)$ discontinued therapy prior to end of study with $50(41.7 \%)$ remaining until conclusion. Of the 82 veterans, in the venlafaxine group, that did achieve therapeutic dosing, 51 (62.2\%) discontinued therapy prior to end of study with $31(37.8 \%)$ remaining on venlafaxine therapy. Our second primary outcome was time to therapeutic dose to investigate the length of time reasonable to expect patients to wait prior to achieving the dose considered evidenced-based for their neuropathic pain. While there were several outliers in both groups remaining on subtherapeutic doses for nearly two years or more, the median time to therapeutic dose for duloxetine was 7 days with interquartile range of 44.3 days compared to median with venlafaxine 31.5 days and interquartile range of 105 days. It was somewhat surprising that both medications were titrated to therapeutic doses so quickly considering they are 
antidepressants. In evaluating our primary outcomes, veterans treated with duloxetine were more likely to achieve therapeutic dose, be titrated quickly, and continue therapy all of which achieved statistical significance. This is perhaps more impressive, considering duloxetine was a restricted formulary medication requiring alternatives to be trialed before it could be approved for use. 117/151 duloxetine patients had tried venlafaxine first per formulary prior to initiating therapy with duloxetine. 35/117 (29.1\%) discontinued venlafaxine after one prescription. Considering that duloxetine patients were significantly older and demonstrated sensitivity to these medications with previous failure of venlafaxine, the results clearly support duloxetine as more tolerable with a higher percentage of patients both achieving therapeutic doses and continuing therapy.

Table 2 Reasons for discontinuation and side effects

\begin{tabular}{|c|c|c|c|}
\hline $\mathrm{N}=302$ & $\begin{array}{l}\text { Venlafaxine } \\
\text { (15I) }\end{array}$ & Duloxetine (15I) & p-value \\
\hline D/c or expired & N (\%) & N (\%) & \\
\hline \multicolumn{4}{|l|}{ Reason: } \\
\hline SE & $65(43.1 \%)$ & $48(31.8 \%)$ & 0.0569 \\
\hline Transferred & $18(11.9 \%)$ & $9(6.0 \%)$ & 0.1052 \\
\hline Lack of efficacy & $23(15.2 \%)$ & $17(1 \mid .3 \%)$ & 0.3962 \\
\hline Non-compliance & $8(5.3 \%)$ & II (7.3\%) & 0.6366 \\
\hline Unknown & $5(3.3 \%)$ & $14(9.3 \%)$ & 0.0554 \\
\hline $\begin{array}{l}\text { Decline in renal } \\
\text { function }\end{array}$ & $\mathrm{I}(0.7 \%)$ & 0 & $\mathrm{I}$ \\
\hline Pain Controlled & 0 & $2(1.3 \%)$ & 0.4983 \\
\hline Total & 120 & 101 & \\
\hline
\end{tabular}

\begin{tabular}{|c|c|c|c|}
\hline \multicolumn{4}{|l|}{ Types of SE: } \\
\hline Change in mood & $20(\mid 3.3 \%)$ & $14(9.3 \%)$ & 0.3629 \\
\hline GI distress & $7(4.6 \%)$ & $7(4.6 \%)$ & I \\
\hline $\begin{array}{l}\text { Sexual } \\
\text { dysfunction }\end{array}$ & $6(4.0 \%)$ & $4(2.7 \%)$ & 0.7497 \\
\hline Sedation & $4(2.7 \%)$ & $4(2.7 \%)$ & I \\
\hline Nightmares & $4(2.7 \%)$ & $2(1.3 \%)$ & 0.6844 \\
\hline SI & $\mathrm{I}(0.7 \%)$ & $\mathrm{I}(0.7 \%)$ & I \\
\hline Hyperhidrosis & $2(1.3 \%)$ & $\mathrm{I}(0.7 \%)$ & I \\
\hline Elevated LFTs & 0 & $\mathrm{I}(0.7 \%)$ & I \\
\hline $\begin{array}{l}\text { Heart } \\
\text { palpitations }\end{array}$ & $\mathrm{I}(0.7 \%)$ & $\mathrm{I}(0.7 \%)$ & I \\
\hline Allergic reaction & $3(2.0 \%)$ & $4(2.7 \%)$ & $\mathrm{I}$ \\
\hline Tremor & $2(1.3 \%)$ & $\mathrm{I}(0.7 \%)$ & I \\
\hline $\begin{array}{l}\text { Urinary } \\
\text { Retention }\end{array}$ & 0 & $3(2.0 \%)$ & 0.2475 \\
\hline Myalgias & $2(1.3 \%)$ & $\mathrm{I}(0.7 \%)$ & I \\
\hline Syncope & $3(2.0 \%)$ & 0 & 0.2475 \\
\hline Dizziness & $2(1.3 \%)$ & $\mathrm{I}(0.7 \%)$ & I \\
\hline Hypertension & $2(1.3 \%)$ & 0 & 0.4983 \\
\hline Weight gain & $I(0.7 \%)$ & $\mathrm{I}(0.7 \%)$ & I \\
\hline Insomnia & $\mathrm{I}(0.7 \%)$ & $\mathrm{I}(0.7 \%)$ & I \\
\hline Bruxism & $\mathrm{I}(0.7 \%)$ & 0 & I \\
\hline Headaches & $3(2.0 \%)$ & 0 & 0.2475 \\
\hline $\begin{array}{l}\text { Serotonin } \\
\text { syndrome }\end{array}$ & 0 & $\mathrm{I}(0.7 \%)$ & I \\
\hline Total & 65 & 48 & \\
\hline
\end{tabular}

Table 3 Concomitant diseases and medications

\begin{tabular}{llll}
\hline $\begin{array}{l}\text { Secondary } \\
\text { endpoints }\end{array}$ & $\begin{array}{l}\text { Duloxetine } \\
\text { I5 I (\%) }\end{array}$ & Venlafaxine I5 I (\%) & P-value \\
\hline Comorbidities & & & \\
\hline Diabetes & $43(28.48)$ & $27(17.88)$ & 0.0403 \\
PTSD & $63(41.72)$ & $70(46.36)$ & 0.4868 \\
Depression & IIO (72.85) & $90(59.60)$ & 0.0206 \\
Anxiety & $53(35.1)$ & $43(28.48)$ & 0.266 \\
\hline Concomitant & medications & & \\
\hline SSRIs & $12(7.95)$ & $17(11.26)$ & 0.435 I \\
SNRIs & $5(3.31)$ & I (0.66) & 0.214 I \\
MAOIs & $0(0)$ & $0(0)$ & I \\
TCAs & $72(47.68)$ & $9(5.96)$ & 0.000 I \\
Gabapentin & $73(48.34)$ & $56(37.09)$ & 0.0625 \\
Pregabalin & $24(15.89)$ & $15(9.93)$ & 0.1692 \\
\hline
\end{tabular}

We identified several secondary outcomes of interest including dropout rate between duloxetine and venlafaxine. Duloxetine matched its predicted rate of discontinuation with previous clinical trials with $20 \%$ failing to achieve therapeutic dosing. In contrast, venlafaxine's rate of discontinuation was more than double that of clinical trials with $45.7 \%$ failing to achieve a therapeutic dose. Another outcome of interest was how rate of titration influenced discontinuation rate (i.e. tolerability). Duloxetine was titrated to therapeutic doses quickly (median 7 days) as expected but venlafaxine was titrated much quicker than expected (median 31 days) with $29.3 \%$ achieving therapeutic doses within 14 days and $47.6 \%$ within 30 days. The discontinuation rate with such a rapid titration was nearly $85 \%$ and did not decrease to $50 \%$ unless titrated more gradually reaching therapeutic doses after 60 days. Unfortunately, comparisons of these secondary outcomes were not sufficiently powered to evaluate significance.

There were several limitations to our study, which include its retrospective design, reliance on provider documentation for inclusion and limited external validity, as majority of patients were white males. Another limitation was due to formulary restriction, most if not all patients in the duloxetine group had to previously try and fail venlafaxine. As the patients in the duloxetine group had already not achieved benefit from a previous SNRI trial, they may be considered higher risk for a second medication failure. And furthermore, the study population was found to have a high rate of psychiatric comorbidities that if mental health was not well managed may have placed those patients at a higher risk for dropout and non-compliance.

\section{Conclusion}

This therapeutic impact of medication selection (TIMS) evaluation showed that in real world conditions, patients started on duloxetine for neuropathic pain were significantly more likely to achieve therapeutic dosing, be titrated to therapeutic dose much quicker, and remain on duloxetine long-term compared to venlafaxine. Based upon these results, the impact of titration schedule may play a role in discontinuation rate but requires further study to evaluate for statistical significance. We further conclude that these results indicate 
that duloxetine should be favored in treatment algorithms over venlafaxine for neuropathic pain in formulary considerations or risk introduction of ethical concerns into treatment.

\section{Acknowledgement}

No grants or funding were associated with this project.

\section{Conflict of interest}

The authors report no conflict of interest.

\section{References}

1. Rudroju N, Bansal D, Talakokkula ST, et al. Comparative efficacy and safety of six antidepressants and anticonvulsants in painful diabetic neuropathy: a network meta-analysis. Pain Physician. 2013;16(6):E705E714.

2. Attal N, Crucca G, Baron R, et al. EFNS guidelines on the pharmacological treatment of neuropathic pain: 2010 revision. Eur $J$ Neurol. 2010;17(9):1113-1123.

3. Global Industry Analysts, Inc. Report, January 10, 2011 http://www.prweb. com/pdfdownload/ 8052240.pdf.

4. Bril V, England J, Franklin GM, et al. Evidence-based guidelines: Treatment of painful diabetic neuropathy: report of the American Academy of Neurology, the American Association of Neuromuscular and Electrodiagnostic Medicine, and the American Academy of Physical Medicine and Rehabilitation. Neurology. 2011;76(20):1785-1765.

5. Brainin M, Barnes M, Baron JC, et al. Guidance for the preparation of neurological management guidelines by EFNS scientific task forcesrevised recommendations 2004. Eur J Neurol. 2004;11(9):577-581.
6. Saarto T, Wiffen PJ. Antidepressants for neuropathic pain. Cochrane Database Syst Rev. 2007;4:CD005454.

7. Finnerup NB, Otto M, McQuay HJ, et al. Algorithm for neuropathic pain treatment: an evidence based proposal. Pain. 2005;118(3):289-305.

8. Max MB, Lynch SA, Muir J, et al. Effects of desipramine, amitriptyline, and fluoxetine on pain in diabetic neuropathy. $N$ Engl $J$ Med. 1992;326(19):1250-1256.

9. Kajdasz DK, Iyengar S, Desaiah D, et al. Duloxetine for the management of diabetic peripheral neuropathic pain: evidence-based findings from post hoc analysis of three multicenter, randomized, double-blind, placebocontrolled, parallel-group studies. Clin Ther. 2007;29 Supp1:2536-2546.

10. Quilici S, Chancellor J, Lothgren M, et al. Meta-analysis of duloxetine versus pregabalin and gabapentin in the treatment of diabetic peripheral neuropathic pain. BMC Neurol. 2009;9:6.

11. Kadiroglu AK, Sit D, Kayabasi H, et al. The effect of venlafaxine $\mathrm{HCl}$ on painful peripheral diabetic neuropathy in patients with type 2 diabetes mellitus. J Diabetes Complications. 2008;22(4):241-245.

12. Stahl SM, Grady MM, Moret C, et al. SNRIs: their pharmacology, clinical efficacy, and tolerability in comparison with other classes of antidepressants. CNS Spectr. 2005;10(9):732-747.

13. Rowbotham MC, Goli V, Kunz NR, et al. Venlafaxine extended release in the treatment of painful diabetic neuropathy: a double-blind, placebocontrolled study. Pain. 2004;110(3):697-706.

14. Pritchett YL, Mc Carberg BH, Watkin JG, et al. Duloxetine for the management of diabetic peripheral neuropathic pain: response profile. Pain Med. 2007;8(5):397-409. 